\section{MARIE SKLODOWSKA-CURIE, SCIENTIFIQUE ET FEMME TOUT SIMPLEMENT}

\author{
Pr. Armand Lattes \\ Professeur émérite à l'Université Paul Sabatier \\ (Toulouse, France)
}

A quoi rêvait Maria Sklodowska pendant le long voyage qu'elle effectuait de Varsovie à Paris, en ces derniers jours d'octobre 1891. Assise sur une banquette d'un wagon de troisième classe au départ de Varsovie, ou sur un pliant au centre d'un wagon de quatrième clase pendant la traversée de l'Allemagne, de nombreuses pensées ont dû accompagner ces trois jours d'un parcours éprouvant.

C'est d'abord vers son pays, la Pologne, que devait s'égarer son esprit. Une fois encore dépecée et partagée entre la Russie, l'Autriche et la Prusse, considérée par les russes -qui en occupaient la plus grande partie- comme une province où la langue n'était pour eux qu'un patois, elle abritait un peuple révolté, mais écrasé, et dont les libertés essentielles avaient été bafouées. Maria avait souffert de cette situation: ainsi, malgré ses remarquables succès scolaires couronnés à 16 ans, en 1886, par le diplôme de fin d'études secondaires avec la mention Très Bien dans toutes les matières, accompagné de la médaille d'or, il lui était interdit, comme à toutes les polonaises, de poursuivre des études supérieures dans son pays. Comme d'autres intellectuelles étrangères, Maria avait choisi de partir étudier à Paris.

C'est sans doute à cette capitale française où reposaient tous ses espoirs, que songeait aussi Maria, cette ville lumière où régnait cette liberté qui fait tant défaut aux polonais, où l'on peut parler librement, dans toutes les langues, et étudier dans une université prestigieuse: la Sorbonne.

Maria arriva suffisamment tôt à Paris pour s'inscrire à cette université dont les premiers cours débutaient le 3 Novembre. C'est en francisant son prénom en Marie qu'el- le rédigea sa feuille d'inscription en licence ès sciences physiques dont elle commença à suivre régulièrement les cours. De tout temps elle avait été attirée par cette discipline, influencée sans doute par son entourage familial et les enseignements qu'elle avait suivis à "I'Université Volante", structure clandestine polonaise où l'on dispensait, en cachette, un enseignement aux jeunes polonaises. Son grand père paternel, Jozef, était, entre autre, professeur de sciences physiques; son père, Wladislaw, avait enseigné les mathématiques, la physique et les sciences naturelles dans des lycées de Varsovie. Ajoutons à ceux-ci ses autres parents scientifiques: son cousin, Edmund Sklodowski, qui avait étudié la chimie à la Sorbonne dans le laboratoire d'Henri Etienne Sainte Claire Deville, et le neveu de sa mère, Jozef Jerzy Boguski, ancien assistant de Dimitri Mendeleiev, qui, après avoir été Professeur de Lycée à Varsovie, avait pris la direction du laboratoire de Physique au Muséum de l'Industrie et de l'Agriculture de cette ville. Boguski qui, par la suite, enseigna dans une école technique et à l'Ecole Polytechnique de Varsovie, permit à Maria d'accéder aux différents laboratoires du Muséum où elle put acquérir des connaissances en physique, mais aussi en chimie grâce à Napoléon Milicer, directeur du laboratoire de chimie et ancien élève de Robert Bunsen (à l'origine des méthodes expérimentales de l'analyse spectrale).

Malgré cette influence et cette formation les débuts à la Sorbonne furent difficiles car Marie ne maîtrisait pas encore parfaitement le français et présentait de grosses lacunes dans ses connaissances. Grâce à un travail acharné tous ces handicaps furent levés et, en 1893, Marie fut reçue première à la licence ès sciences physiques avec la mention très bien, puis, en 1894, à la licence de mathématiques, deuxième de sa promotion, avec la mention assez bien. 
Mais comment une jeune polonaise, issue d'un milieu intellectuel mais modeste, avait-elle pu trouver les moyens matériels de se rendre à Paris et de quoi vivait-elle dans cette capitale? Cela débuta par un contrat moral avec sa sœur Bronia: celle-ci rêvait de faire des études de médecine à Paris, mais pour ce faire il lui fallait disposer d'une somme suffisante pour son voyage, ses inscriptions, ses dépenses quotidiennes. Maria lui proposa de l'aider en lui envoyant l'argent qu'elle pourrait gagner en se plaçant comme institutrice dans une famille. Réciproquement, Bronia devrait aider Maria quand elle sera docteur en médecine. C'est ainsi que, le contrat étant rempli, Marie rejoignit Bronia et son mari, le docteur Casimir Dluski, dans leur logement de la rue d'Allemagne, près du parc des Buttes Chaumont, à la Villette.

Le couple Dluski travaille dur... mais aime aussi beaucoup bavarder, se distraire, recevoir, jouer du piano, toutes occupations sympathiques mais qui génèrent des nuisances peu compatibles avec le désir de Marie de travailler en paix! De plus, il fallait au moins une heure en omnibus hippomobile pour rejoindre la Sorbonne avec, en supplément, une dépense élevée en frais de transport. Marie décida alors d'habiter près des écoles et s'installa dans une petite chambre, au loyer peu élevé, au $6^{\text {ème }}$ étage d'un immeuble du quartier latin: "froide en hiver", "brûlante en été". Avec 40 roubles par mois soit 3 francs par jour, on ne peut vivre que pauvrement, et il arriva que Marie, en mal de nourriture, s'évanouisse, suscitant la crainte de son beau-frère et de sa sœur.

C'est alors qu'une amie polonaise, Melle Dydynska, mathématicienne, prend l'initiative de la faire présenter pour l'attribution de la bourse Alexandrowitch (soit 600 roubles), bourse destinée à des étudiants polonais peu fortunés qui méritaient de continuer à étudier à l'étranger. Une fois obtenue cette bourse, en 1893, ce fut, pour Marie, 15 mois de subsistance assurés sans souci matériel majeur.

\section{Rencontre avec Pierre Curie}

Pendant la préparation de la licence ès sciences mathématiques, une proposition intéressante devait améliorer le quotidien de Marie: la Société d'Encouragement pour

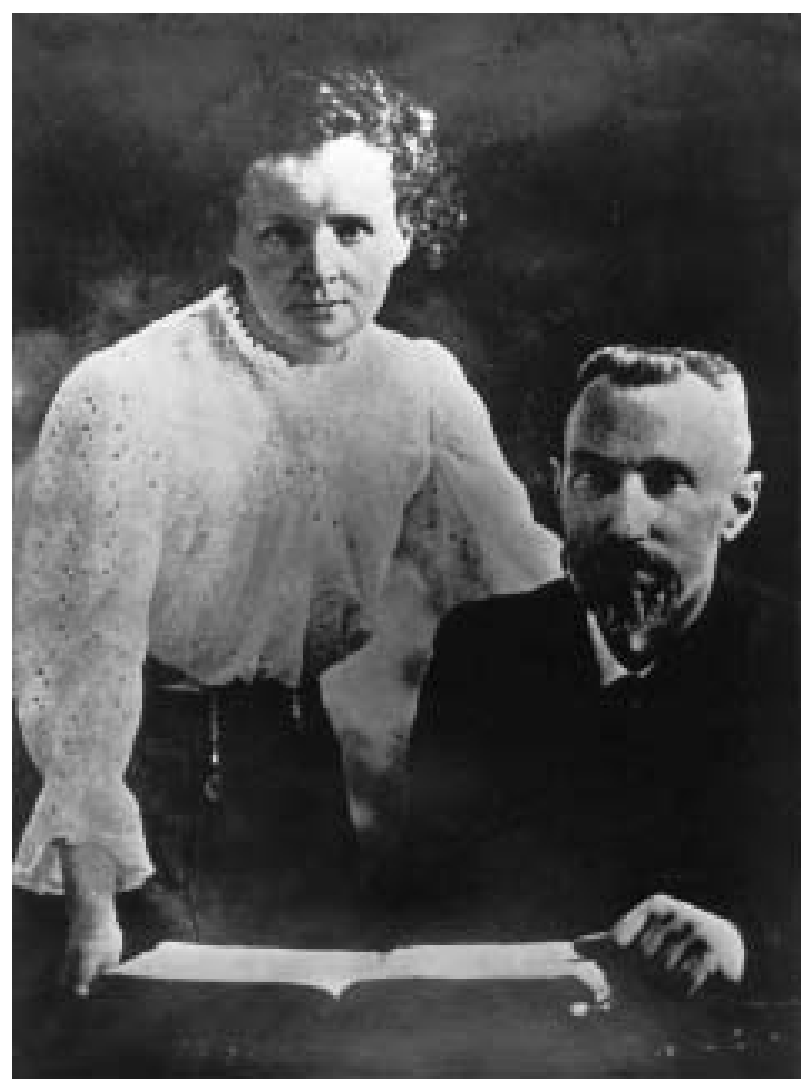

I'Industrie Nationale, lui commanda une étude expérimentale sur les propriétés magnétiques de divers types d'acier. C'est dans le laboratoire du Professeur Lippmann qu'elle commença ce travail, mais très vite les échantillons de minerais, des métaux, devinrent trop encombrants et il devint nécessaire de chercher un nouveau local pour continuer les manipulations. Ayant exposé ses difficultés à un compatriote physicien, le Professeur Jozef WiernzKowalski, enseignant à Fribourg, celui-ci a une idée qui va changer totalement le cours de sa vie. II connaît un jeune physicien à qui demander conseil: Pierre Curie, chef de travaux à l'Ecole Municipale de Physique et de Chimie Industrielles de Paris, déjà connu pour ses travaux sur le magnétisme et la cristallographie. C'est au cours d'une soirée dans la chambre de la pension de famille où habitaient les Kowalski pendant leur séjour parisien, que Marie fit la connaissance de Pierre Curie.

Dès leur première rencontre Pierre Curie est séduit! Lui qui avait écrit dans son journal: ... "les femmes de génie 
sont rares" est surpris par cette polonaise qui est capable de comprendre ses travaux et même de les discuter. II souhaite la revoir et commence une cour de moins en moins discrète.

Marie, elle, bien qu'attirée par Pierre, n'a pas répondu favorablement à sa demande en mariage. Sans doute porte-t-elle encore en son cœur les stigmates d'un amour polonais déçu! Sans doute aussi se sent-elle obligée de servir son pays et donc de retourner en Pologne. Ces mois d'été 1894, elle s'éloigne en Suisse puis en Pologne. Mais Pierre insiste et lui écrit cette si belle phrase:

"Ce serait cependant une belle chose à laquelle je n'ose croire, que de passer la vie l'un près de l'autre, hypnotisés dans nos rêves: votre rêve patriotique, notre rêve humanitaire et notre rêve scientifique"

(un rêve qui ne devait durer que 10 ans d'une collaboration intense)

Marie revient à Paris en octobre 1894, Pierre continue sa cour et... après 10 mois, elle consent à l'épouser: ce qui fut fait le 26 juillet 1895; un mariage civil, sans anneau ni prière pour cet agnostique et cette catholique qui avait cessé de pratiquer. Sa belle famille est adorable: le Docteur Curie, père de Pierre et sa femme l'accueillirent avec affection.

Commença alors pour elle une vie très différente de celle qu'elle avait menée jusqu'alors. Le logement des jeunes mariés étant tout près de l'Ecole de Physique et Chimie, la vie devenait plus facile pour Marie qui fut autorisée par le directeur de cette école, le chimiste Paul Schützenberger, à continuer son travail sur les aciers dans cet établissement où travaillait Pierre. Leur souhait de vivre toujours ensemble est exaucé, exaucé aussi le vœu de Pierre:

"Il faut faire de la vie un rêve, et faire d'un rêve une réalité"

C'est ensemble encore qu'ils se promenaient en bicyclette tout autour de Paris, en Auvergne, dans les Cévennes ou à Brest.Elle n'est plus seule et s'applique à exercer du mieux possible sa condition d'épouse: elle fait le marché, apprend à cuisiner, annote des recettes... mais toujours rien ne compte davantage que le travail... En 1896, elle se présente au concours de l'Agrégation de Physique où elle est reçue première. Son travail sur les aciers, qu'elle mène en parallèle, est terminé en 1897 et fait l'objet d'un article: "Les propriétés magnétiques des aciers trempés" publié au Bulletin de la Société d'Encouragement à I'Industrie Nationale en 1898.

Au printemps 1897, Marie Curie est enceinte et, le 12 septembre de cette année, Irène vient au monde. Cette naissance bouscule les projets de Marie, mais ne l'éloigne pas de sa décision de continuer à travailler. Au décès de sa belle mère, son beau-père, le docteur Curie, vient habiter avec Pierre et Marie dans leur nouveau pavillon: il sera une aide précieuse pour l'éducation d'Irène à laquelle il est très attaché.

\section{LA DÉCOUVERTE}

Décidée à préparer un doctorat, Marie s'interroge quelque temps sur le choix du thème de recherche: les récents travaux d'Henri Becquerel sur les rayonnements émis spontanément par les sels d'Uranium attirent sa curiosité.... Quelle est la nature de ces rayons? C'est décidé, son sujet de thèse sera: "I'étude des rayons uraniques"

Après une expérience réalisée fin février, début mars 1896, Becquerel avait énoncé cette conclusion "L'Uranium et ses sels émettent spontanément des rayons pénétrants" II s'agissait seulement d'une étude qualitative, Marie Curie se proposa de profiter des propriétés d'ionisation de l'air par ces rayons pour effectuer des mesures quantitatives du phénomène. Pour cela elle allait utiliser des appareils résultant des découvertes de Jacques et Pierre Curie:

- le quartz piézoélectrique, dont l'étirement ou la compression génère des charges électriques (sources étalons de charges);

- l'électromètre à quadrants qui, couplé au quartz piézoélectrique, permet de mesurer des courants extrêmement faibles.

Disposant de l'électromètre à quartz, Marie Curie entreprit une étude systématique de la conductibilité de l'air que provoquent ces rayons. Les résultats, publiés en 
avril 1898, confirment que les composés contenant de l'uranium émettent des rayonnements... mais c'est également une propriété que possède le thorium! Ils montrent aussi que le rayonnement est une propriété de l'élément Uranium et que son intensité est proportionnelle à la quantité d'uranium présente quelque soit la nature du sel, indépendamment de toute circonstance extérieure, température ou lumière. Le Thorium présentant les mêmes propriétés, mais avec une intensité différente de celle de l'Uranium, on ne pouvait plus parler de "rayons uraniques", et Marie Curie invente et utilise, dans une publication du 18 juillet 1898, pour la première fois, le mot Radioactivité.

Examinant divers échantillons de sels et de minerais d'Uranium, Marie Curie constate alors que deux minerais, la pechblende et la chalcolite, présentent une activité beaucoup plus élevée que ne le laisserait prévoir leur teneur en Uranium. Au contraire, une chalcolite pure, de synthèse, présente une activité en accord avec cette teneur. Marie Curie en déduisit que les minerais naturels devaient contenir au moins une substance inconnue, un élément nouveau, très fortement radioactif. Cette observation remarquable où on montre l'existence d'un élément alors qu'il n'a été ni isolé, ni même observé, incite Pierre à rejoindre totalement Marie pour des travaux qu'ils vont désormais mener en commun.

Leurs premières recherches conduisirent le couple à isoler une substance, proche du Bismuth par ses propriétés chimiques, environ 400 fois plus radioactive que I'Uranium, substance qu'ils décidèrent d'un commun accord d'appeler Polonium.

Au cours de ces travaux, un autre élément, différent par ses propriétés chimiques du Polonium (proches, cette fois, des propriétés du Baryum) est également découvert, simplement caractérisé par analyse spectroscopique, élément auquel les Curie donnèrent le nom de Radium. Cette découverte fut publiée dans les Comptes Rendus de l'Académie des Sciences en Décembre 1898. Mais... I'analyse spectrale n'était pas suffisante pour faire valider ce nouvel élément: il devenait nécessaire de préparer une quantité suffisante de Radium pour le caractériser et déterminer sa masse atomique. C'est alors que commence une odyssée extraordinaire qui montre le caractère, la volonté et le courage de Marie Curie.
Les résidus de Pechblende, après extraction de l'Uranium, contenaient toujours du Polonium et du radium: c'est sur ce matériel, et dans des conditions très difficiles, que Marie appliqua sa technique de séparation. Des tonnes de résidus furent traités pour qu'enfin, en 1902, 1 décigramme de radium fut isolé, tandis que la masse atomique de cet élément fut déterminée, soit $225 \pm 1$ (ce qui est toujours aujourd'hui, la valeur retenue).

Le 25 juin de cette même année 1902, Marie Curie soutint sa thèse devant un Jury de la faculté des Sciences de Paris, avec comme titre: "Recherches sur les substances radioactives"; ce jury devait compter par la suite 3 prix Nobel: Gabriel Lippmann, Président, Prix Nobel de Physique 1908; Henri Moissan, Prix Nobel de Chimie 1906; et, bien sûr Marie Curie 2 fois prix Nobel. Sa soutenance fut couronnée par le grade de Docteur ès Sciences Physiques avec la mention Très Honorable: c'était la première fois, en France, qu'une femme obtenait ce grade.

De nombreuses récompenses accompagnèrent cette Recherche de qualité: 3 fois le prix Gegner pour Marie, la médaille Davy pour le couple et enfin, en 1903, le Prix Nobel de Physique décerné pour moitié à H.Becquerel, l'autre moitié revenant à Pierre et Marie. Ces derniers avaient été proposés, dès 1901, par le Professeur Charles-Jacques Bouchard, mais, en 1903, un groupe d'Académiciens Français proposa Becquerel et Pierre Curie, sans mentionner Marie! Pierre, informé par Gösta Mittag-Leffler, mathématicien suédois, écrivit à ce dernier:

"... dans le cas où il serait vrai que l'on songe sérieusement à moi, je désirerais beaucoup que l'on me considère comme solidaire avec Mme Curie dans nos recherches sur les corps radioactifs"

En raison d'un état de santé très mauvais, Pierre et Marie ne purent se rendre à Stockholm pour la cérémonie, et c'est seulement en 1905 qu'ils eurent la possibilité de le faire.

\section{La mort de Pierre Curie}

Cette reconnaissance scientifique améliora considérablement le quotidien de la famille Curie: tandis que Pierre devenait chargé de cours à la Sorbonne, en 1900, Marie 
fut, la même année, chargée de conférences de Physique à l'Ecole Normale Supérieure de jeunes filles à Sèvres. En 1904, Pierre était nommé Professeur dans une nouvelle chaire à la Sorbonne, et Marie chef de travaux de Physique, rattachée à la chaire de son mari. Leurs découvertes, par contre, ne leur rapportèrent rien car ils avaient refusé de breveter leur procédé de préparation des sels de Radium; Marie Curie écrira plus tard:

"le radium ne doit enrichir personne: c'est un élément, il appartient à tout le monde "

Leur vie familiale continuait son cours, modestement; après la naissance d'Irène, en 1897, la mort à la naissance d'une deuxième fille en 1903, le 6 décembre 1904 Marie Curie donna le jour à sa fille Eve. Très amoureux l'un de l'autre, le couple partageait la même passion de la Recherche, malheureusement un horrible accident vint interrompre cette collaboration et cette belle harmonie: Pierre Curie, le 19 avril 1906, fut écrasé par un camion hippomobile. Ce fut un choc terrible pour Marie qui écrivit dans son journal, 11 jours après la mort de Pierre:

"Cher Pierre que je ne verrai plus jamais ici, je veux te parler dans le silence de ce laboratoire où je n'aurais jamais pensé devoir vivre sans toi"

Avec son courage habituel, elle allait reprendre ses activités et succéder à son époux dans la chaire de celui-ci à la Sorbonne: elle devenait ainsi la première femme à devenir professeur de faculté en France.Sa leçon inaugurale fut un événement mondain: le 5 novembre 1906, dans un amphithéâtre bondé, toute vêtue de noir elle commença son cours en prononçant ces quelques mots:

"Lorsqu'on envisage les progrès qui ont été accomplis en Physique depuis une dizaine d'années, on est surpris du mouvement qui s'est produit dans les idées sur l'électricité et la matière"

Puis elle continua en reprenant le cours là où son mari l'avait laissé.

Continuant ses recherches dans le but d'obtenir du chlorure de Radium pur, puis du Radium métallique, c'est en 1910, en collaboration avec Debierne, qu'elle atteignit son but, mesurant ainsi le point de fusion du métal: $700^{\circ} \mathrm{C}$. La même année, avec le même collaborateur, ils établirent le caractère élémentaire du Polonium par l'observation des raies spectrales à partir d'un dixième de milligramme de matériel. Lors des discussions sur l'adoption de l'étalon international du Radium, I'unité de mesure fut baptisée Curie et sa définition fut imposée par Marie: "la quantité d'émanation en équilibre avec 1 gramme de Radium"

L'admiration que suscitait l'exceptionnelle scientifique se traduisit par une avalanche de titres, récompenses, et honneurs. Académicienne de nombreuses institutions étrangères, Marie Curie se laissa convaincre par nombre de scientifiques de se présenter, en janvier 1911, au siège occupé peu de temps par son mari à l'Académie des Sciences Française. Plus ou moins bien reçue par les académiciens, Marie fut battue le 23 janvier par Edouard Branly, à une courte majorité: 30 voix contre 28! Henri Poincaré, un de ses partisans, s'exprima ainsi "elle sera reçue la prochaine fois". Déçue, Marie Curie décida de ne plus jamais se présenter.

\section{Le Scandale et le prix Nobel de Chimie}

Cette élection avait été précédée d'une campagne de presse où les journaux prenaient parti, dans un sens ou un autre quant à sa candidature; l'opposition se manifestant surtout dans la presse de droite. Ces débats et ce tapage eurent un effet dévastateur: la vie privée de Marie, que jusqu'alors elle avait préservée avec succès, fut brutalement livrée en pâture aux lecteurs avides de scandales.

Le 4 Novembre 1911, le journal, publiait un article intitulé: "une histoire d'amour: Mme Curie et le Professeur Langevin". Reprises le lendemain par tous les journaux parisiens, puis par la presse internationale, ces informations atteignirent Marie Curie à Bruxelles où elle participait au congrès Solvay. Malgré les demandes de rectificatifs corrigeant les mensonges publiés par des journaux sans scrupules, malgré les menaces de poursuites judiciaires, la campagne continua et, le 23 Novembre, l'œuvre, petite brochure hebdomadaire, publia sous le titre "Les scandales de la Sorbonne" des extraits des lettres de Marie à Paul Langevin. Ces extraits, choisis et séparés du reste 
de la correspondance, ne montrent que peu de chose: l'avis de Marie sur l'union de Langevin avec sa femme, ses conseils quant à la conduite qu'il devait adopter, et l'aveu d'une complicité intellectuelle très forte et d'une solide amitié. Comme l'écrivit André Langevin, le fils de Paul, dans son livre "Paul Langevin, mon père: I'homme et l'œuvre":

"N'est-il pas assez naturel que cette amitié, doublée d'une admiration mutuelle, se soit, plusieurs années après la mort de Pierre Curie, transformée en passion puis en liaison?"

Veuve depuis 5 ans, âgée seulement de 44 ans en 1911, elle était victime des conventions de l'époque où le coupable de ces situations était toujours la femme! De nos jours cela se serait borné à des commentaires privés et ne serait jamais allé jusqu'à un tel déchaînement de passions malsaines. Malgré son désarroi et son état de santé, Marie Curie fit front avec son courage habituel. Entre temps après son retour à Paris, le 8 novembre 1911, un télégramme venant de Stockholm l'informait que le prix Nobel de Chimie lui était attribué.

Après une longue réflexion sur l'attitude à adopter, c'est avec sa sœur Bronia et sa fille Irène qu"elle se rendit à Stockholm le 11 décembre pour recevoir sa récompense. Elle prononça le lendemain le discours de réception: "Le Radium et les nouveaux concepts en chimie", avec force et dignité, acclamée par toutes les personnes présentes, revendiquant sa part des découvertes qui lui valaient cette dignité, mais rendant hommage à la mémoire de Pierre Curie en rappelant que "le travail chimique qui avait pour but d'isoler le radium à l'état pur... se trouve entièrement lié à l'œuvre commune"

Tous ces événements, tous ces efforts, laissèrent Marie Curie dans un état de santé déplorable et, le 29 décembre, elle fut emmenée sur une civière dans une maison de repos. En janvier 1912, elle rentra chez elle, guérie,mais encore faible, et on dût attendre avril 1912 avant de l'opérer d'un rein. De nouveau déprimée et épuisée, ce n'est qu'après un séjour dans un sanatorium à Thonon, puis en Angleterre chez Mrs Hertha AYRTON, physicienne et féministe qui fut pour beaucoup dans sa guérison, que le 17 octobre 1912 elle reprit sa place dans son bureau de la rue Cuvier.

\section{LA POURSUITE DES TRAVAUX ET LA CRÉATION DE L'INSTITUT DU RADIUM}

Pendant tout ce temps à partir d'observations de H.Becquerel et P. Curie qui avaient expérimenté sur euxmêmes les effets physiologiques du Radium, I'utilisation des émissions de cet élément pour le traitement du cancer commençait à s'imposer. Des Instituts du Radium étaient créés dans de nombreux pays européens et, grâce aux efforts de Marie, en 1912 I'Institut Pasteur et I'Université de Paris conclurent un accord qui aboutit à la fondation, à Paris, de l'Institut du Radium qui comprenait 2 composantes:

- l'une, que dirigeait Marie Curie, pour la physique et la chimie,

- l'autre, dirigée par le Docteur Claude REGAUD, pour la recherche médicale et biologique.

A la fin du mois de juillet 1914, I'Institut fut achevé, mais le 2 Août, la $1^{\text {ère }}$ guerre mondiale éclatait. Devant l'avance allemande Marie Curie transporta son gramme de radium à Bordeaux où elle le confia à la Faculté des Sciences.

\section{Les Petites Curie}

En 1915, Marie Curie s'installa dans son laboratoire du nouveau bâtiment, rue Pierre Curie, mais souhaitant s'engager le mieux possible auprès de l'armée française, répondant aussi aux vœux d'Irène qui souhaitait se rendre utile, elle offrit de mettre ses compétences à la disposition du service de santé des armées. C'est ainsi qu'elle fut chargée de former des équipes de manipulateurs en radiologie.

En peu de temps, nommée officiellement directeur du service de radiologie de la Croix Rouge, elle réussit à mettre en service plus de 200 voitures (appelées les petites Curie!) dont les équipements de radiologie permettaient d'aider les chirurgiens dans leur recherche de la localisation des balles et éclats d'obus dans les plaies des blessés, et d'opérer dans les meilleures conditions. Avec sa fille Irène, âgée seulement de 18 ans, elle participa activement à de nombreux examens se déplaçant d'hôpital à hôpital, au volant d'une ambulance, au service des blessés. 
Mais son action ne se limitait pas à cela: Marie Curie utilisa son nouvel Institut du Radium pour y former des manipulatrices en radiologie. Ayant connaissance des propriétés remarquables du Radon (émanation du Radium), source de rayons traitants, elle créa le premier service de thérapie au Radium qui fournissait aux hôpitaux du Radon enfermé dans de minces tubes qui étaient ensuite placés aux endroits du corps où ils étaient les plus efficaces, grâce à des aiguilles de platine.

Après l'armistice, Marie Curie réintégra son nouveau laboratoire et fut confronté très vite à des problèmes matériels pour le fonctionnement de son équipe de recherche. Elle qui rêvait de créer une Ecole de la Radioactivité, devait mendier des crédits pour pouvoir continuer son travail! C'est alors qu'en mai 1920 elle fit une rencontre qui devait lui apporter une solution.

\section{L'AVENTURE AMÉRICAINE}

La rédactrice en chef du journal américain "The Delineator", Marie Mattingley-Meloney, surnommée Missy par ses amis, avait sollicité une interview auprès de Marie Curie. Celle-ci, marquée par l'affaire Langevin, ne recevait pas de journalistes sauf pour parler sciences et techniques. L'écrivain français Henri-Pierre Roché, auteur de Jules et Jim, intervint auprès d'elle pour qu'elle accepte de recevoir Missy. Les deux femmes, bien que très différentes l'une de l'autre dans leur comportement, vont sympathiser et rester amies.

Missy ne devait pas se contenter de rédiger un article biographique, ayant demandé à Marie:

"Si vous pouvez formuler un vœu, que souhaiteriez-vous posséder?"

celle-ci lui répondit:

"j'aurais besoin d'un gramme de Radium afin de poursuivre mes recherches, mais je ne peux l'acheter, le Radium est trop cher pour moi"

Missy se proposa alors de sensibiliser les américains à ces problèmes. De retour aux USA, elle organisa une campagne nationale pour recueillir les 100000 dollars nécessaires à cet achat. En quelques mois elle réussit, et cela au delà de ses espérances puisque, une fois le Radium acheté, il resta 50000 dollars!

Après accord de l'Université de Paris, Marie et ses deux filles (23 et 16 ans) embarquèrent sur l'Olympic de la White Star Line, le 4 mai 1921. A I'arrivée à New York, le 11 mai, une foule immense l'attendait tandis qu'une meute de journalistes l'interviewait sur le pont du paquebot. Le 20 mai, le Président des USA, Warren Gamaliel Harding, lui remit un coffret d'acajou, doublé de plomb, pesant une cinquantaine de kilos, et renfermant un gramme de Radium, dont elle avait fait entériner officiellement le fait qu'elle pourrait l'utiliser librement et sans contrainte à des fins expérimentales et pour le progrès de la science. Le reste de l'argent recueilli permettait aussi à Marie d'équiper son laboratoire et de faire fonctionner son équipe.

Plus tard, Missy continua à trouver de nouveaux crédits: plus spécialement, elle orientait les américaines fortunées, visitant Paris, vers le bureau de la rue Pierre Curie où Marie recevait le mardi et le vendredi et acceptait les dons qu'elles lui apportaient.

\section{LES DERNIĖRES ANNÉES ET LES DERNIERS TRAVAUX}

Le voyage aux USA avait été épuisant pour Marie qui avait dû courir de cérémonies en cérémonies. Son retour à Paris fut plus discret et c'est avec soulagement qu'elle retrouva son laboratoire de I'Institut du Radium où elle forma de nombreux scientifiques, dont sa fille Irène et le futur époux de celle-ci: Frédéric Joliot. Sa fille qui, 20 ans après sa mère, soutenait sa thèse en Sorbonne, thèse qu'elle lui dédia ainsi:

"A madame Curie, sa fille et son élève".

Marie Curie continua ses travaux jusqu'à la fin de sa vie. De 1920 à 1934, elle développa essentiellement des études sur la chimie des éléments radioactifs. Ainsi, elle prépara des quantités importantes de Polonium dont elle étudia les propriétés chimiques et grâce auquel elle suivit l'émission des particules á. Elle devait contribuer, avec Salomon Rosenblum, à l'étude de la structure fine de ces rayons á qui devait permettre de poser la base expérimentale précise de l'existence de différents niveaux énergétiques pour 
un noyau atomique. Ses efforts se portèrent aussi sur la préparation de produits actinifères, sources radioactives, et sur l'ionium, un isotope du thorium. Grâce aux sources intenses de Polonium, Irène et Frédéric Joliot devaient découvrir la radioactivité artificielle.

Les conséquences des découvertes de Marie Curie sont considérables! Elle faisait sienne les paroles de Pierre Curie prononcées à Stockholm lors de la cérémonie Nobel:

"Je suis de ceux qui pensent avec Nobel que l'Humanité tirera plus de bien que de mal des découvertes nouvelles"

Outre la découverte et la caractérisation physico-chimique de deux éléments, le Polonium et le Radium, elle est à l'origine de la création de la science de la Radioactivité qui permit de découvrir la structure de l'atome et du noyau atomique, et de la mise en évidence de la fission grâce à laquelle on a pu exploiter l'énergie nucléaire.

Refusant de croire que ses apports scientifiques pouvaient être dangereux, elle mourut d'une leucémie, le 4 juillet 1934, au sanatorium de Sancellemoz, en Savoie, victime de ses manipulations multiples de substances radioactives.
Inhumée le 6 juillet, dans le caveau de famille aux côtés de Pierre, au cimetière de Sceaux, ses cendres et celles de son époux ont été transférées au Panthéon, le 20 avril 1995, en présence de sa fille Eve.

Cette femme extraordinaire, au destin exceptionnel, a montré toute sa vie un courage et une détermination exemplaires. Son caractère apparaît dans sa maxime qui montre sa détermination:

"Dans la vie rien n'est à craindre, tout est à comprendre"

Française et Polonaise elle n'a jamais oublié son pays d'origine auquel elle a apporté son assistance scientifique en aidant à la création d'établissements disposant des mêmes innovations que celles développées en France à partir de ses travaux.

D'une modestie rare, le plus beau compliment qu'elle mérite est, sans conteste, celui que prononça à son intention Albert Einstein:

"Marie Curie est, de tous les êtres célèbres, le seul que la gloire n'ait pas corrompu" 